\title{
Análise via TRI da Escala Baptista de Depressão Infanto-Juvenil e do Inventário de Depressão Infantil
}

\author{
Makilim N. Baptista ${ }^{1}$ \\ Nelson Hauck Filho ${ }^{1}$ \\ Fernanda Grendene ${ }^{2}$ \\ ${ }^{1}$ Universidade São Francisco, SP, Brasil \\ ${ }^{2}$ Universidade Regional Integrada do Alto Uruguai e das Missões, Campus Erechim, RS, Brasil
}

\begin{abstract}
Resumo
O presente estudo teve como objetivo analisar as propriedades psicométricas de dois instrumentos avaliativos de sintomatologia depressiva, o Inventário de Depressão Infantil (CDI) e a Escala Baptista de Depressão Infanto-Juvenil (EBADEP-IJ), nas versões completa e short. Além da análise dos parâmetros de dificuldade e discriminação dos itens, foram comparadas as curvas de informação do CDI e da EBADEP-IJ, a fim de avaliar o nível de precisão de cada instrumento ao longo do contínuo latente da depressão. A análise de 331 respostas de crianças e adolescentes revelou que os dois instrumentos possuem adequadas propriedades psicométricas, embora com uma ligeira diferença no que diz respeito à capacidade de diferenciar indivíduos localizados em regiões distintas da variável latente. Os achados sustentam o uso de ambos os instrumentos em pesquisas na área, e também apontam para possíveis usos diferenciados para cada instrumento.
\end{abstract}

Palavras-chave: Sintomas depressivos; Crianças; Adolescentes; Teoria de Resposta ao Item.

\section{Analysis of IRT of the Child-Juvenile Baptista Depression Scale and of the Children's Depression Inventory}

\begin{abstract}
This study reports on a psychometric appraisal of two self-report instruments developed to assess depressive symptoms, namely the Children's Depression Inventory (CDI) and the Baptista's Depression Scale - Youth Version (EBADEP-IJ). Besides performing an analysis of difficulty and discrimination item parameters, we compared the test information curves from both instruments as a means to have a more in-depth assessment of precision along the latent continuum of depression. Analysis of data from 331 children and adolescents revealed that both instruments have sound psychometric properties, despite they are slightly different as to latent trait coverage. The findings support the use of both CDI and EBADEP-IJ for research purposes, and they suggest different specific uses for each inventory.
\end{abstract}

Keywords: Depressive symptoms; Children; Adolescents; Item Response Theory.

\section{Análisis de la TRÍ de la Escala Baptista de Depresión Infanto Juvenil y del Inventario de Depresión Infantil}

Resumen

Este estudio tuvo como objetivo analizar las propiedades psicométricas de dos instrumentos de evaluación de síntomas depresivos, Inventario de Depresión Infantil (CDI) y los niños y jóvenes Escala de Depresión Baptista (EBADEP-IJ), las versiones completas y cortos. En el análisis de los parámetros de dificultad y discriminación de los ítems, en comparación con las curvas de información CDI y EBADEP-IJ, para evaluar el nivel de precisión de cada instrumento sobre la depresión continua latente. Los resultados de 331 respuestas de los niños y adolescentes revelaron que los dos instrumentos son propiedades psicométricas adecuadas aunque con una ligera diferencia con respecto a la capacidad para diferenciar los individuos situados en diferentes regiones de la variable latente. Estos resultados apoyan el uso de ambos instrumentos en la investigación en el área, y también apuntan a posibles usos diferentes para cada instrumento.

Palabras clave: Síntomas depresivos; Niños; Adolescentes; Teoría de Respuesta al Ítem. 


\section{Introdução}

A depressão é um transtorno de humor e/ou afetivo que pode acometer sujeitos de todas as faixas etárias. É um problema complexo que se origina da interação de fatores psicológicos, socioambientais e biológicos, sendo considerada atualmente uma das patologias incapacitantes mais prevalentes no mundo (World Health Organization, 2012). Em crianças e adolescentes, os sintomas de depressão podem se manifestar de diversas formas, tais como demonstração de tristeza, desmotivação, irritabilidade, negativismo, sentimentos de inutilidade, comportamentos agressivos, prédisposição para brigas, alterações de sono, mudanças no rendimento escolar, queixas somáticas e mudanças de apetite. Os sintomas podem ser similares à manifestação da depressão em adultos, embora a idade module as características e as repercussões negativas do transtorno (Mendéz, Olivares, \& Ros, 2005). Além disso, a presença de um Episódio Depressivo Maior na infância ou adolescência pode afetar diferentes funções, tanto cognitivas quanto afetivas e picossociais que incapacitam o funcionamento do indivíduo (American Psyquiatric Association, APA, 2014).

Com relação ao diagnóstico da depressão na infância e adolescência, é importante investigar minuciosamente as diferentes variáveis relacionadas a ela, com intuito de se obter um diagnóstico preciso da doença, uma vez que a mesma pode estar associada a diferentes fatores, tais como os psicológicos, físicos e sociais (Wilkinson, 2009). Nesse sentido, são necessários instrumentos de avaliação psicológica que auxiliem no diagnóstico preciso da depressão na infância e adolescência. O presente estudo aborda a temática da avaliação psicológica da depressão nestas faixas etárias, investigando e comparando a capacidade informativa de dois instrumentos de auto relato, a Escala Baptista de Depressão Infanto-Juvenil (Baptista \& Cremasco, 2013), nas versões completa e short e o Inventário de Depressão Infantil, versão adaptada por Gouveia, Barbosa, Almeida e Gaião (1995).

O CDI é um instrumento consolidado internacionalmente e utilizado para avaliar a sintomatologia depressiva na infância e adolescência (Cruvinel \& Boruchovitch, 2008). Foi criado nos Estados Unidos, em 1985, por Kovacs, e provém de uma adaptação do Inventário de Depressão de Beck (BDI). Trata-se de um instrumento de auto relato do tipo Likert que em sua versão original contém 27 itens. Serve para mensurar sintomas depressivos em crianças e adolescentes de sete a 17 anos. No Brasil, o CDI foi adaptado por Gouveia et al. (1995), com uma amostra de 305 crianças e adolescentes de 8 a 15 anos do estado da Paraíba, sendo o alfa de Cronbach de 0,81, com estrutura unifatorial e o instrumento final composto por 20 questões distribuídas entre os sintomas afetivos, cognitivos e comportamentais.

Com o objetivo de verificar as propriedades psicométricas do CDI, outros estudos ainda utilizaram versões adaptadas do instrumento para o contexto Brasileiro. Golfeto, Veiga, Souza e Bandeira (2002) aplicaram o CDI, adaptado por Gouveia et al. (1995), em 287 escolares de 7 a 14 anos de Ribeirão Preto. Foram encontrados, por meio de análise de componentes principais, cinco componentes que explicaram $47,4 \%$ da variância total. Nesse estudo, porém os autores consideraram mais adequado assumir sua estrutura unifatorial do CDI, uma vez que a consistência interna de cada fator foi considerada baixa (menor que 0,70 ). Os autores também encontraram um alfa de Cronbach de 0,81. Já Coutinho, Carolino e Medeiros (2008) da mesma forma, estudaram a estrutura interna do CDI com 280 escolares, com idades variando entre 9 e 17 anos e encontraram uma solução unifatorial $(36,86 \%$ da variância total), representada por 20 itens além de índice de consistência interna de 0,91. Ainda, Coutinho, Oliveira e Pereira (2014) avaliaram 730 estudantes da cidade de Teresina - Piaú, com idades entre 9 e 17 anos, com o objetivo de adaptar e verificar evidências de validade de construto e consistência interna do CDI. Efetuou-se uma análise fatorial, e os resultados indicaram uma solução unifatorial composta por 17 itens. Essa estrutura explicou $22,56 \%$ da variância total, com um índice de consistência interna alfa de Cronbach de 0,82. Além desses estudos Vega et.al (2016), avaliaram as propriedades psicométricas de uma versão reduzida de 10 itens do CDI, o CDI-S, em crianças e adolescentes com deficiência física. Os resultados indicaram em consonância com outros estudos uma solução de um fator, com um índice de consistência interna alfa de Cronbach de 0,84.

Com relação à escala Baptista de Depressão InfantoJuvenil (EBADEP-IJ) (Baptista \& Cremasco, 2013), o instrumento também investiga a sintomatologia depressiva em crianças. Tal instrumento utiliza os mesmos descritores da EBADEP versão para adultos, com itens baseados em descritores presentes em manuais psiquiátricos, como o DSM-IV-TR (American Psychiatric Association, 2002) e CID-10 (Organização Mundial da Saúde, 1993) além de indicadores presentes na Cognitiva da Depressão (Beck, Rush, Shaw, \& Emery, 1982). Também foram levados em conta Princípios do Comportamento (Ferster, Culbertson, \& Boren, 1977) e descritores baseados em Weinberg, Rutman, Sullivan, Pencik e Dietz (1973). A versão da EBADEP-IJ é adequada à faixa de oito a 18 anos. 
Um dos diferenciais da escala é a divisão dos itens entre positivos e negativos, que permite a avaliação da sintomatologia depressiva também por itens de respostas positivas tais como "tenho planos para o futuro e gosto de mim como sou". A inclusão de tais componentes vai ao encontro da perspectiva atual de que a saúde mental só pode ser compreendida a partir da totalidade de seus aspectos positivos e negativos (Machado \& Bandeira, 2015).

Baptista e Cremasco (2013) estudaram evidências de validade baseada na relação com variáveis externas para a EBADEP-IJ, em uma amostra com 241 escolares sendo $167(69,3 \%)$ do sexo feminino, com idades entre 8 e 17 anos de escalas públicas de uma cidade do interior do Estado de São Paulo. Os instrumentos utilizados, além da EBADEP-IJ, foram o Inventário de Percepção do Suporte Familiar (IPSF), a Escala de Percepção do Suporte Social (EPSUS) e Inventário de Depressão Infantil (CDI) como medida auxiliar. Com relação aos resultados do CDI correlacionados com a EBADEP-IJ e aos demais instrumentos, foram encontradas correlações similares entre os dois e as demais variáveis estudadas. Tanto o CDI quanto a EBADEP-IJ apresentaram correlações negativas com as dimensões do IPSF, do IPSF Total e da EPSUS.

No estudo realizado por Leite(2011), que objetivou a análise fatorial exploratória da EBADEP-IJ, a comparação de escores de grupo depressivo com grupo não depressivo e a correlação dos escores da escala com os escores de diversos outros instrumentos, participaram da amostra 697 adolescentes entre 11 e 17 anos. Dentre as correlações encontradas observaramse, altas magnitudes entre os instrumentos EBADEP-IJ e o CDI, $r=0,80(p<0,001)$.

Tanto o CDI quanto a EBADEP-IJ são instrumentos com aceitáveis propriedades psicométricas que se propõem a avaliar a sintomatologia depressiva, porém ainda são necessários estudos que também avaliem quão bem os itens dos testes cobrem as dimensões latentes. Um estudo de Baptista, Hauck e Cardoso (2016) no qual buscou-se analisar a relação entre os constructos teóricos depressão e bem-estar subjetivo, a partir de uma amostra de crianças e adolescentes, utilizou o CDI e a EBADEP-IJ, além da EBES. Os resultados da análise bifatorial realizada apontam para características fatoriais das escalas aqui discutidas, CDI e EBADEP-IJ, como a de o CDI apresentar cargas positivas em um fator geral de psicopatologia (aspectos negativos) e a da EBADEP - IJ apresentar cargas positivas tanto com o fator de psicopatologia (aspectos negativos), quanto com um fator específico, de aspectos positivos. Frente à necessidade de avaliar as escalas, os modelos de Análise da Teoria de Resposta ao Item (TRI) são particularmente úteis para investigar em que nível do traço latente cada instrumento funciona, ou seja, com que precisão ao longo do continuum depressão e em que região cada um deles tem maior funcionalidade. A curva de informação dos testes é uma vantagem da TRI em relação à teoria clássica dos testes (Kamata $\&$ Bauer, 2008). Além disso, a TRI é de extrema relevância na análise de traços latentes, pois propicia um maior aproveitamento da informação, trabalhando não somente com a classificação dos indivíduos quanto ao traço latente, como também trazendo informações sobre os instrumentos de medida como um todo e, principalmente, item a item (Castro, Trentini, \& Reboldi, 2010). Tais dados psicométricos podem ser úteis para que o clínico/pesquisador possa ter informações de quando um instrumento pode ser mais adequado na utilização quando comparado a outro, o que justifica a necessidade de mais estudos direcionados a precisão, discriminação e funcionamento dos itens.

Dentro deste contexto, alguns estudos utilizaram-se da TRI para avaliar os itens de instrumentos que avaliam depressão em idosos e adultos. Com o objetivo de verificar quais itens da Escala de Depressão Geriátrica (EDG) são mais discriminativos para detectar depressão em idosos saudáveis e demenciados, Pereira et al. (2012) analisaram os escores de 15 itens a partir de prontuários de 149 pacientes idosos saudáveis e demenciados. Dos 15 itens, 8 tiveram valores maiores que 1 no parâmetro $a$, mostrando-se bastante discriminativos . Os autores concluíram que uma versão EDG-8 pode ser um substituto para a EDG-15 por apresentar apenas os itens mais discri-minativos, separando de maneira mais eficiente os pacientes deprimidos numa triagem clínica. Neste sentido, alguns autores (Lam, Michalak, \& Swinson, 2006; Nezu, Ronan, Meadows, \& McClure, 2000) também referem que versões reduzidas são úteis para rastrear sintomas em estudos epidemiológicos, em avaliações psicológicas breves, para avaliar a eficácia terapêutica ou quando necessita-se de uma bateria extensa de testes.

Castro et al. (2010) avaliaram a Escala Beck de Depressão analisada via TRI e o resultado da análise paralela mostrou que a suposição de unidimensionalidade pode ser considerada suficiente pois foi encontrado um fator preponderante com $38,7 \%$ da explicação da variância total. Os autores ainda referem que os indivíduos que responderam o BDI não conseguiram distinguir algumas afirmações nas categorias de resposta de determinados itens, mostrando a necessidade de se repensar o dimensionamento da escala. Tal problema apareceu em treze itens, entre os quais dois que se referem a sintomas que carregam uma grande quantidade de informação psicométrica sobre a intensidade de 
sintomas depressivos, pessimismo e insatisfações. A solução apresentada no estudo foi juntar as categorias de resposta adjacentes àquela com problema, pois é provável que os indivíduos não tenham distinguido os conteúdos das afirmações contidas nestas categorias.

Nesta perspectiva, no presente estudo foram investigados os parâmetros psicométricos (dificuldade, discriminação e funcionamento das categorias) dos itens do CDI e da EBADEP-IJ e comparadas as curvas de informação dos dois instrumentos, incluindo uma versão reduzida da EBADEP-IJ, denominada Short a fim de avaliar o nível de precisão de cada instrumento ao longo do contínuo latente da sintomatologia depressiva.

\section{Método}

\section{Participantes}

Participaram do estudo 331 crianças e adolescentes, selecionados por conveniência, cujas idades variaram de 10 a 16 anos $(M=12,42 ; D P=1,48)$, sendo $53,8 \%$ do sexo feminino. Todos os sujeitos eram alunos do ensino fundamental ou médio de escolas públicas e particulares da cidade de São Paulo.

\section{Instrumentos}

Escala Baptista de Depressão Infanto-juvenil (EBADEP-IJ) (Baptista \& Cremasco, 2013): É uma escala que visa identificar a sintomatologia depressiva em crianças e adolescentes de oito a 18 anos. É composta por 45 itens que investigam 23 descritores: humor deprimido; perda ou diminuição de prazer; choro; desesperança/falta de perspectiva sobre o futuro; desamparo; indecisão; sentimento de incapacidade e inadequação; carência/dependência; negativismo; esquiva de situações sociais; queda de produtividade; inutilidade; autocrítica exacerbada; culpa; diminuição da concentração; pensamento de morte; autoestima rebaixada; falta de perspectiva sobre o presente; hipocondria; alteração de apetite; alteração de peso; alteração de sono (insônia/hipersonia).

A escala é do tipo Likert, na qual cada item sugere uma situação que é respondida de três possíveis formas, Nunca/Poucas Vezes, Algumas vezes e Muitas Vezes/ Sempre. A pontuação máxima alcançada na escala é de 90 pontos, sendo atribuídos pontos da seguinte forma: zero para os itens assinalados como "Nunca"; um ponto aos itens assinalados "Algumas Vezes" e dois pontos a última opção restante. Quanto maior a pontuação maior a sintomatologia depressiva. A consistência interna das subescalas obtida no estudo original apresentou índices aceitáveis, sendo que as medidas do alfa variaram entre 0,86 e 0,89 , enquanto o lambda de Guttman variou em 0,87 e 0,91 (Baptista \& Cremasco, 2013).
Escala Baptista de Depressão Infanto-Juvenil (EBADEP-IJ) - versão Short. A EBADEP-IJ-Short foi desenvolvida por Baptista (2015), e consiste em apenas 15 itens. Foram escolhidos aqueles que são mais descritos nos manuais psiquiátricos considerados como "core symptoms", que são eles: $1,2,8,10,12$, 13, 14, 19, 20, 26, 29, 32, 33, 36 e 38.

Inventário de Depressão Infantil (Children's Depression Inventory ou CDI). O CDI foi construído por Kovacs (1985), a partir de uma adaptação do Inventário de Depressão de Beck (BDI), e tem como objetivo mensurar sintomas depressivos em jovens com idade entre sete e dezessete anos. No presente estudo, foi utilizada a versão brasileira adaptada por Gouveia et al. (1995), composta por 20 itens. Cada item possui três alternativas de resposta variando entre: 0 (ausência de sintoma), 1 (presença de sintoma) e 2 pontos (sintoma grave). O respondente é instruído a assinalar a opção que melhor descreve seu estado nas últimas duas semanas. Dessa forma, quanto maior for sua pontuação, maior será sua sintomatologia depressiva. O teste é autoaplicado, no qual os respondentes assinalam uma das três possíveis alternativas de cada item, a melhor que descreve seus sentimentos nas duas últimas semanas. Nesta versão foi adotada uma solução unifatorial, sendo o alfa de Cronbach de 0,81 .

\section{Procedimentos}

A coleta de dados foi realizada após a autorização do Comitê de Ética em Pesquisa da Universidade São Francisco e posteriormente pelas instituições de ensino onde foram realizadas as coletas. Os instrumentos foram aplicados de forma coletiva e em situação de sala de aula, sendo que participaram somente os alunos cujos pais assinaram o Termo de Consentimento Livre e Esclarecido (TCLE). As crianças e adolescentes que não tiveram a aprovação dos pais foram direcionadas pelos professores a outras atividades em outro ambiente escolar diferente da sala onde ocorria a aplicação dos instrumentos. A ordem de aplicação dos instrumentos seguiu de forma alternada para cada turma, buscando minimizar um possível efeito fadiga sobre os resultados dos testes com um tempo aproximado de aplicação de $1 \mathrm{~h}$.

\section{Análise dos dados}

Os dados foram analisados por meio de técnicas descritivas e inferenciais. As propriedades psicométricas dos instrumentos EBADEP-IJ e CDI foram avaliadas utilizando o modelo de Teoria de Resposta ao Item Graded Response Model (GRM; Samejima, 1969). Esse modelo permite estimar os parâmetros dos thresholds (dificuldades de endosso das categorias) e da discriminação dos itens, sendo o equivalente a um 
modelo de dois parâmetros logísticos (2-PL), embora para itens de natureza politômica em vez de dicotômica.

Como o modelo GRM assume a unidimensionalidade dos itens, esse pressuposto foi, primeiramente, testado a partir de uma análise bi-factor exploratória de cada instrumento. Reise, Morizot e Hays (2007) indicaram o teste de um modelo bi-factor como uma maneira eficaz de avaliar a unidimensionalidade de um conjunto de dados. Essa análise foi feita utilizando o estimador Unweighted Least Squares (ULS) e uma matriz de correlações policóricas, procedimento recomendado como ideal quando as variáveis são de natureza ordinal (Asún, Rdz-Navarro, \& Alvarado, 2016), como no presente caso (escala Likert). Subsequentemente, foram estimados os parâmetros do modelo GRM e a capacidade informativa de cada teste por meio das curvas de informação dos testes, que possibilitam entender a região do traço latente em que o teste fornece escores com maior nível de precisão. As análises foram conduzidas por meio dos pacotes ltm (Rizopoulos, 2006) e psych (Revelle, 2014) do programa R.

\section{Resultados}

Inicialmente, testou-se o pressuposto da unidimensionalidade dizer se é de ambos os dados por meio da análise fatorial exploratória bi-factor. Para tanto, empregou-se a função "omega" do pacote psych (Revelle, 2014). Os resultados da análise com a EBADEP-IJ revelaram coeficientes ômega $=0,96$ e alpha $=0,95$, sendo que 41 dos itens carregaram acima de 0,20 (entre 0,21 e 0,64) em um fator geral de depressão. Itens positivos carregaram em um fator específico de aspectos saudáveis, e itens negativos carregaram em outro fator de aspectos patológicos. No caso do instrumento CDI, os coeficientes ômega e alpha foram ambos 0,92 , e todos os itens carregaram apenas em um fator geral de depressão, com cargas fatoriais variando entre 0,21 e 0,81 . Esses resultados foram considerados evidência de unidimensionalidade, tanto no CDI como na EBADEP-IJ, com uma variável latente principal explicando os dados, sustentando então o uso do modelo GRM.

Na sequência, foram realizadas as análises de TRI com cada um dos instrumentos. Como a capacidade informativa de um instrumento ocorre em função do número de itens, e existe uma grande desigualdade entre EBADEP-IJ e CDI no que diz respeito a esse aspecto (43 versus 20 itens). Na versão final da EBADEP-IJ ainda foram incluídos mais 2 itens, totalizando 45 , porém considerou-se nas análises a versão com 43 itens. Também se considerou aqui uma versão reduzida da EBADEP-IJ, a EBADEP-IJ-Short. A EBADEP-IJShort foi desenvolvida por Baptista (2015), e consiste em apenas 15 itens. Foram escolhidos aqueles que são mais descritos nos manuais psiquiátricos considerados como "core symptoms", que são eles: 1, 2, 8, 10, 12, 13, 14, 19, 20, 26, 29, 32, 33, 36 e 38. Destes, os seguintes são itens positivos: 1, 20, 26, 29 e 36, sendo os demais itens negativos.

TABELA 1

Resultados da Análise da EBADEP-IJ com o Graded Response Model.

\begin{tabular}{|c|c|c|c|c|c|c|c|c|c|c|c|c|c|}
\hline \multirow{2}{*}{ Item } & \multicolumn{3}{|c|}{43 itens } & \multicolumn{3}{|c|}{15 itens } & \multirow{2}{*}{ Item } & \multicolumn{3}{|c|}{43 itens } & \multicolumn{3}{|c|}{15 itens } \\
\hline & b1 & $b 2$ & $a$ & bl & $b 2$ & $a$ & & $b 1$ & $b 2$ & $a$ & $b 1$ & $b 2$ & $a$ \\
\hline 1 & $-1,76$ & 6,00 & 0,29 & $-4,15$ & 11,40 & 0,12 & 23 & 1,19 & 3,39 & 0,79 & & & \\
\hline 2 & 0,22 & 0.22 & 1,20 & 0,15 & 1,75 & 1,13 & 24 & 0,12 & 1,68 & 1,48 & & & \\
\hline 3 & 6,26 & 1,99 & 1,49 & & & & 25 & 1,29 & 3,54 & 0,52 & & & \\
\hline 4 & 0,32 & 1,46 & 1,98 & & & & 26 & 0,39 & 1,99 & 1,13 & 0,42 & 2,50 & 0,77 \\
\hline 5 & -1.47 & 0,56 & 0,56 & & & & 27 & 0,60 & 2,41 & 1,27 & & & \\
\hline 6 & 1,14 & 2,60 & 1,42 & & & & 28 & 0,60 & 1,82 & 2,04 & & & \\
\hline 7 & 1,84 & 4,07 & 1.03 & & & & 29 & $-0,99$ & 1,84 & 0,90 & $-1,25$ & 2,12 & 0,71 \\
\hline 8 & 0,38 & 3,12 & 0.70 & 0,30 & 3,07 & 0,69 & 30 & 0,37 & 2,15 & 0,92 & & & \\
\hline 9 & 1,47 & 3,51 & 1,01 & & & & 31 & 0,66 & 2,02 & 1,33 & & & \\
\hline 10 & 0,49 & 2,70 & 1,28 & 0,41 & 2,62 & 1,25 & 32 & 1,25 & 2,21 & 1,56 & 1,04 & 1,92 & 1,84 \\
\hline 11 & 0,97 & 2,29 & 2,03 & & & & 33 & 1,16 & 2,36 & 1,23 & 0.99 & 2.08 & 1.38 \\
\hline 12 & 0,31 & 1,40 & 1.83 & 0,18 & 1,07 & 3,04 & 34 & -0.84 & 1.97 & 0.72 & & & \\
\hline 13 & 0,42 & 1,74 & 1,52 & 0,25 & 1,30 & 2,56 & 35 & 0,52 & 2.61 & 0.82 & & & \\
\hline 14 & 0.62 & 1.84 & 2.21 & 0.48 & 1.62 & 2.60 & 36 & 0.26 & 2.11 & 0.87 & 0.22 & 2.43 & 0.69 \\
\hline 15 & -0.52 & 2.36 & 0.86 & & & & 37 & -0.38 & 1.80 & 0.97 & & & \\
\hline 16 & $-5,28$ & 1,32 & 0,29 & & & & 38 & 0.87 & 3.89 & 0.67 & 0.87 & 4.22 & 0.59 \\
\hline 17 & 0,88 & 6,11 & 0,42 & & & & 39 & -0.91 & 2.05 & 0.80 & & & \\
\hline 18 & $-0,55$ & 1,67 & 0,84 & & & & 40 & -0.36 & 2.44 & 0.80 & & & \\
\hline 19 & 0,63 & 4,75 & 0,48 & 0,43 & 3,66 & 0,63 & 41 & 0.21 & 2.33 & 1.12 & & & \\
\hline 20 & 2,35 & 6,16 & 0,39 & 3,58 & 9,63 & 0,24 & 42 & 0.55 & 1.86 & 1.40 & & & \\
\hline 21 & 0,65 & 1,88 & 1,77 & & & & 43 & 0,62 & 2.05 & 1.43 & & & \\
\hline 22 & 0,88 & 2.28 & 1.35 & & & & & & & & & & \\
\hline
\end{tabular}


TABELA 2

Resultados da análise do CDI com o Graded Response Model.

\begin{tabular}{cccc}
\hline Item & \multicolumn{3}{c}{20 itens } \\
1 & $b 1$ & $b 2$ & $a$ \\
2 & 1,49 & 2,91 & 1,86 \\
3 & 0.06 & 2,79 & 1,85 \\
4 & 1,32 & 3,67 & 5,10 \\
5 & 0,49 & - & 1,11 \\
6 & 2,95 & 7,02 & 0,88 \\
7 & 0.008 & 5,03 & 0,73 \\
8 & 1,20 & 2,53 & 2,15 \\
9 & 1,07 & 2,77 & 1,64 \\
10 & 0,67 & 3,22 & 1,85 \\
11 & 0.90 & 1,93 & 2,66 \\
12 & 1,10 & 2,76 & 1,15 \\
13 & 2.20 & 3,88 & 1,26 \\
14 & 0,30 & 2,58 & 1,12 \\
15 & 0,47 & 3,02 & 1,39 \\
16 & 1,55 & 3,70 & 0,86 \\
17 & 0,64 & 2,03 & 2,19 \\
18 & 0,62 & 3,97 & 1,10 \\
19 & -0.37 & 2,16 & 1,92 \\
20 & 0,98 & 2,97 & 1,48 \\
\hline & 0,07 & 7,54 & 0,41 \\
\hline
\end{tabular}

A Tabela 1 apresenta os parâmetros de discriminação e dificuldade encontrados para os itens das duas versões da EBADEP-IJ (as análises foram conduzidas separadamente para cada versão), enquanto a Tabela 2 apresenta os resultados para o CDI. Os valores $b 1$ e $b 2$ indicam o nível necessário para ter $50 \%$ de probabilidade de endossar, respectivamente, o segundo e o terceiro enunciado que compõem cada item do instrumento. Valores baixos indicam itens menos patológicos, e valores altos indicam itens mais patológicos. Valores de a indicam a capacidade discriminativa dos itens, sendo que quanto mais alto maior a discriminação.

Foram então conduzidas as análises da curva de informação de cada instrumento. Novamente, foram consideradas EBADEP-IJ e EBADEP-IJ-Short, além do CDI. A Figura 1 apresenta o resultado dessas análises, permitindo ajudar a visualizar a área específica avaliada pelo conjunto de itens de cada instrumento. Pontos de informação representam o inverso do quadrado do erro padrão de medida observado para a escala em cada ponto do traço latente. Como esperado, o instrumento EBADEP-IJ, em sua versão de 43 itens, ofereceu a maior quantidade de informação psicométrica dentre os três conjuntos de itens, sendo 76,29 pontos. O gráfico possibilita ver que a EBADEP-IJ parece avaliar melhor os níveis de depressão na faixa theta entre 0 e 2 pontos. Por sua vez, EBADEP-IJ-Short ofereceu um total de 29,58 pontos e o CDI 35,96 pontos, uma pequena diferença justificada pelos cinco itens a mais no CDI em relação à EBADEP-IJ-Short. Uma inspeção do gráfico permite ver que o CDI e as EBADEP-IJ nas duas versões parecem funcionar melhor em regiões ligeiramente distintas do contínuo latente da depressão. Especificamente, enquanto a EBADEP-IJ parece avaliar melhor níveis entre 0 e 2 da escala theta, o CDI parece funcionar melhor um pouco mais à direita da escala, entre 0,5 e 2,5, que significa pessoas com mais depressão.

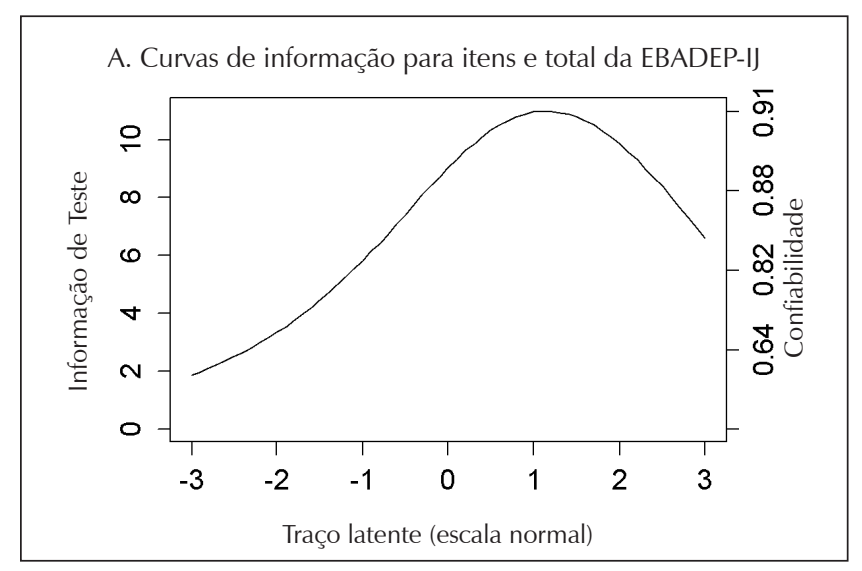

B. Curvas de informação para itens e total da EBADEP-IJ-Short
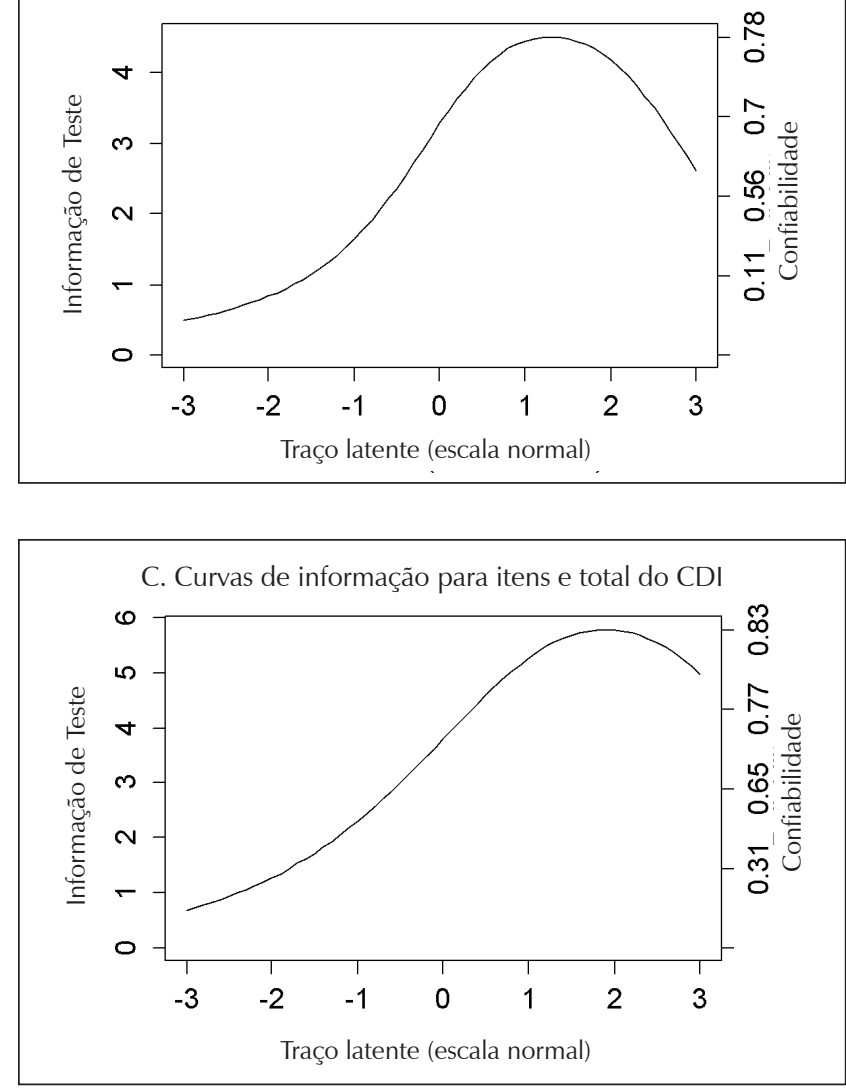

Figura 1. Curvas de informação para itens e total dos testes 


\section{Discussão}

O objetivo do presente estudo foi avaliar as propriedades psicométricas de dois instrumentos avaliativos de sintomatologia depressiva, o CDI e a EBADEP-IJ em suas versões completa e short. Buscou-se investigar os parâmetros psicométricos (dificuldade, discriminação e funcionamento das categorias) dos itens do CDI e da EBADEP-IJ nas suas duas versões, e comparar as curvas de informação de ambos, a fim de avaliar o nível de precisão de cada um ao longo do contínuo latente (Embretson \& Reise, 2000). Os resultados das análises indicaram um bom ajuste para o modelo unidimensional tanto do CDI como da EBADEP-IJ. Ambos os instrumentos apresentaram itens discriminativos (i.e., altos valores para cargas fatoriais e parâmetros $a$ ), o que sugere uma adequada capacidade de diferenciar indivíduos localizados em regiões distintas da variável latente.

Com relação à EBADEP-IJ completa, os itens da escala apresentaram maior quantidade de informação que o CDI e a EBADEP-IJ-Short, ou seja, maior abrangência do contínuo latente. Em parte, essa capacidade se dá pela quantidade de itens da escala que é maior que os outros dois instrumentos. $\mathrm{Na}$ versão reduzida, houve uma diminuição da quantidade de informação proporcionada, ainda que tenha se observado que a EBADEP-IJ-Short é mais informativa quanto à depressão no nível moderado a leve. Do ponto de vista da prática clínica, as escalas (versão completa e short) podem ter seu uso diferenciado em função de disponibilidade de tempo e para o objetivo da avaliação. Ou seja, para uma avaliação compreensiva, a versão original gera mais informação, além de conter mais descritores para fundamentar a tomada de decisão do clínico. Já a versão short pode ser utilizada para rastreio de sintomas depressivos em estudos epidemiológicos, para situações de avaliação breve ou mesmo quando o clínico necessita de avaliar a eficácia terapêutica ou é requerida uma bateria extensa de testes (Nezu et al., 2000).

A capacidade discriminativa da EBADEP-IJ foi comparável à do CDI. Ambos os instrumentos se mostraram boas escolhas para uma avaliação breve de sintomas de depressão. Entretanto, foi também observada uma ligeira diferença no que tange à área do contínuo latente da depressão que ambos os instrumentos avaliam com maior precisão. A EBADEP$\mathrm{IJ}$, dessa forma, pode ser mais adequada do que o CDI para situações de rastreio clínico e/ou situações em que o clínico necessita realizar uma avaliação rápida e informações importantes no manejo clínico. Nesses casos, há maior interesse em ter uma boa capacidade discriminativa exatamente na área em que não é tão explícita a diferenciação entre sintomatologia leve e clinicamente significativa, além do que, como afirmam Lam et al. (2006), avaliações com escalas em versões curtas podem ser bastante úteis para monitorar respostas ao tratamento clínico.

\section{Conclusões}

O presente estudo, além de constituir um teste empírico das propriedades psicométricas dos dois instrumentos, ilustra a utilidade da TRI ao item no refinamento de instrumentos psicométricos para avaliar depressão. A curva de informação dos testes é uma vantagem da TRI em relação à teoria clássica dos testes, e mesmo em relação a modelos de análise fatorial categórica, análogos da TRI (Kamata \& Bauer, 2008). Vale ressaltar ainda que há uma escassez de instrumentos para avaliação de sintomas depressivos em crianças e adolescentes no Brasil. Futuros estudos psicométricos dos instrumentos construídos e/ou adaptados para nossa cultura podem ser de grande valia, no sentido de proporcionar métodos de mensuração desse fenômeno complexo em todas as faixas etárias.

\footnotetext{
Referências

American Psychiatric Association. (2002). Manual diagnóstico e estatístico de transtornos mentais: DSM-IV-TR. Porto Alegre: Artes Médicas.

American Psychiatric Association (APA). (2014). Manual diagnóstico e estatístico de transtornos mentais: DSM (5ª ed.). Porto Alegre: Artmed.

Asún, R. A., Rdz-Navarro, K., \& Alvarado, J. M. (2016). Developing multidimensional Likert scales using item factor analysis: The case of four-point items. Sociological Methods \& Research, 45(1), 109-133. https://doi. org/10.1177/0049124114566716

Baptista, M. N., Hauck F. N., \& Cardoso, C.(2016). Depressão e bem-estar subjetivo em crianças e adolescentes: testes de modelos teóricos. Psico (Porto Alegre), 47(4), 259-267. https://doi.org/10.15448/1980-8623.2016.4.23012
} 
Baptista, M. N. (2015). Escala Baptista de Depressão Infanto-Juvenil - versão Short (EBADEP-IJ-Short). Campinas, SP: Universidade São Francisco. (Versão elaborada para o presente estudo).

Baptista, M. N., \& Cremasco G. (2013). Propriedades psicométricas da Escala Baptista de Depressão Infanto-Juvenil (EBADEP-IJ). Arquivos Brasileiros de Psicologia, 65(2), 198-213.

Beck, A. T., Rush, A. J., Shaw, B. F., \& Emery, G. (1982). Uma visão geral. In A. T. Beck (Ed.), Terapia cognitiva da depressão (pp. 15-45). Rio de Janeiro, RJ: Zahar.

Castro, S. M. J., Trentini, C, \&. Riboldi, J. (2010). Teoria da resposta ao item aplicada ao Inventário de Depressão Beck. Revista Brasileira de Epidemiologia, 13(3), 487-501. https://doi.org/10.1590/S1415-790x20100000300012

Coutinho, M. P. L., Carolino, Z. C. G., \& Medeiros, E. D. (2008). Inventário de Depressão Infantil (CDI): Evidências de validade de constructo e consistência interna. Avaliação Psicológica, 7(3), 291-300.

Coutinho, M. P. L., Oliveira, M. X., \& Pereira, D. R. (2014). Indicadores psicométricos do Inventário de Depressão Infantil em amostra infanto-juvenil. Avaliação Psicológica, 13(2), 269-276.

Cruvinel, M., \& Boruchovitch, E. (2008). Sintomas depressivos em crianças: Estudos com duas versões do CDI. Psicologia: Ciência e Profissão, 28(3), 574-585. https://doi.org/10.1590/S1414-98932008000300011

Embretson, S. E., \& Reise, S. P. (2000). Item response theory for psychologists. Mahwah, New Jersey: Lawrence Earlbaum Associates.

Ferster, C. B., Culbertson, S., \& Boren, M. C. (1977). Depressão clínica. In C. B. Ferster, S. Culbertson, \& M. C. Boren (Eds.). Princípios do comportamento (pp. 699-725). São Paulo: Hucitec.

Gouveia, V. V., Barbosa, G. A., Almeida, H. J. F, \& Gaião, A. A. (1995). Inventário de Depressão Infantil-CDI: Estudo de adaptação com escolares de João Pessoa. Jornal Brasileiro de Psiquiatria, 44(7), 345-349.

Golfeto, J. H., Veiga, M. H., Souza, L., \& Bandeira, C. (2002). Propriedades psicométricas do Inventário de Depressão Infantil (CDI) aplicados em uma amostra de escolares de Ribeirão Preto. Revista Psiquiatria Clinica, 29(2), 66-70.

Kamata, A., \& Bauer, D. J. (2008). A Note on the relation between factor analytic and item response theory models. Structural Equation Modeling, 15(1), 136-153. https://doi.org/10.1080/10705510701758406

Lam, R. W., Michalak, E. E., \& Swinson, R. P. (2006). Why use assessment scales in clinical practice? In R. W. Lam, E. E. Michalak, \& R. P. Swinson (Orgs.). Assessment scales in depression and anxiety (pp. 1-3). Ashford, UK: CRC Press.

Leite, C. A. (2011). Estudos preliminares da Escala de Depressão Infanto-Juvenil (Dissertação de mestrado). Programa de Pós-Graduação Stricto Sensu em Psicologia, Universidade São Francisco, São Francisco.

Machado, W.L \& Bandeira, D.R. (2015).Positive Mental Health Scale: Validation of the Mental Health ContimuumShort Form. Psico-USF, Bragança Paulista, 20(2), 259-274. https://doi.org/10.1590/1413-82712015200207

Mendéz, F. X., Olivares, J., \& Ros, M. C. (2005). Características clínicas e tratamento da depressão na infância e adolescência. In V. E. Caballo \& M. Á. Simón (Orgs.). Manual de psicologia clínica infantil e do adolescente: Transtornos especificos (pp. 139-185). Santos, SP: Santos.

Nezu, A. M., Ronan, G. F., Meadows, E. A., \& McClure, K. S. (2000). A 10-step guide to selecting assessment measures in clinical and research settings. In A. M. Nezu, G. F. Ronan, E. A. Meadows, \& K. S. McClure (Eds.), Practitioner's guide to empirically based measures of depression (pp. 17-24). New York: Kluwer Academics/Plenum Publishers.

Organização Mundial da Saúde. (1993). Classificação de transtornos mentais e de comportamento da CID-10. Porto Alegre. Artmed.

Pereira, D. A., Velozo F., Lakhdari, F., Giglio, A. G., Melchiades, A., Silva, S. L. da, \& Tomaz, C. (2012). Redução da Escala de Depressão Geriátrica (EDG) pela Teoria de Resposta ao Item. Trabalho apresentado no XVIII Congresso de Geriatria e Gerontologia, Rio de Janeiro, RJ. Resumo recuperado de https://ibneuro.org/docs/ ReducaoEDG_TRI.pdf

Reise, S. P., Morizot, J., \& Hays, R. D. (2007). The role of the bifactor model in resolving dimensionality issues in health outcomes measures. Quality of Life Research, 16(1), 19-31. https://doi.org/10.1007/s11136-007-9183-7

Revelle, W. (2014). Psych: Procedures for personality and psychological research. Evanston, Illinois: Northwestern University, R package version.

Rizopoulos, D. (2006). 1tm: An R package for latent variable modeling and item response theory analyses. Journal of Statistical Software, 17(5), 1-25. https://doi.org/10.18637/jss.v017.i05

Samejima, F. (1969). Estimation of latent ability using a response pattern of graded scores. Psychometrika Monograph Supplement, 34(4, Pt. 2), 1-100. https://doi.org/10.1007/BF02290599

Vega, R., Racine, M., Rodriguez S.E., Solé, E., Castarlenas, E., Jensen P. M., Engel, J. \& Miró. J. (2016). Psychometric properties of the short form of the Children's Depression Inventory (CDI-S) in young people with physical disabilities. Journal de Psycosomatic Research, 90, 57-61. https://doi.org/10.1016/j.jpsychores.2016.09.007

Weinberg, W. A., Rutman, J., Sullivan, L., Pencik, E. C., \& Dietz, S. G. (1973). Depression in children referred to an education diagnostic center. Journal of Pediatrics, 83, 1065-1072. https://doi.org/10.1016/S0022-3476(73) 80552-9 
Wilkinson, P. (2009). Conceptualization about internalizing problems in children and adolescents. Ciência \& Saúde Coletiva, 14(2), 373-381. https://doi.org/10.1590/S1413-81232009000200007

World Health Organization (WHO). (2012). Depression. A Global Public Health Concern. Department of Mental Health and Substance Abuse. Recuperado de: http://who.int/mental_health/management/depression/who_paper_depression_ wfmh_2012.pdf

\section{Conflitos de interesse:}

Os autores declaram não possuírem conflitos de interesse.

Dados dos autores:

Makilim N. Baptista - Doutor, Universidade São Francisco.

Nelson Hauck Filho - Doutor, Universidade São Francisco.

Fernanda Grendene - Doutora, Universidade Regional Integrada do Alto Uruguai e das Missões.

Endereço para correspondência:

Nelson Hauck Filho

Universidade São Francisco, SwiFt

Rua Waldemar Cesar da Silveira, 105 - Vila Cura D'Ars

13045-510 Campinas, São Paulo, Brasil

$<$ hauck.nf@gmail.com>

Recebido em: 13.03.2017

Aceito em: 08.04.2018 